\title{
A BeiDou Navigation System Aided Method for Localization in Manually Deployed Wireless Sensor Networks
}

\author{
Ping Sun, Zhe Li, Jian-Yi Peng, Wen-Quan Zhang \\ Department of Optoelectronic Equipment, Wuhan Ordnance Non-Commissioned Officer Academy, \\ Wuhan, Hubei 430075, China \\ E-mail: sp_9046@163.com
}

\begin{abstract}
BeiDou Navigation System can provide PNT services for mainland China. In this paper we present the design, implementation and evaluation of a simple, practical and cost effective Localization solution for wireless sensor network notes. This method is designed especially for manual deployments of WSN. We evaluate the solution exclusively in real deployments of BeiDou models. Experiments results show that $100 \%$ of the deployed notes have been successfully located and that the average errors are within 2 meters.
\end{abstract}

Keywords-WSN; beidou navigation system; location

\section{INTRODUCTION}

Notes in Wireless Sensor Networks (WSN) need position information to complete their missions such as target detection and area control. These notes always possess limited resources (e.g. memory, processing power, radio bandwidth, energy) and this kind of limitation should be dealt with when design the WSNs [1-2]. China's BeiDou navigation system now can provide regional navigation services for users throughout Asia. The advanced status of the space segment and the recent release of COMPASS/BeiDou-2-capable multi-constellation GNSS receivers by US and European manufacturers result in an increased public awareness and attention of the scientific community [3]. As a result, solutions based on BeiDou can be an alternative method for WSNs positioning problems. Accurate localization without sophisticated, expensive hardware is a problem that remains unsolved. In this paper we present a solution to manual deployed WSNs notes.

Sensor networks, driven by both industrial and academic developments, make location-awareness one of the essential services for many emerging applications. Many researches have been proposed to address the localization problem in sensor networks. Some of them are designed under certain assumptions and evaluated in simulation environments. For example, the Amorphous positioning algorithm proposed in [4] uses offline hop-distance estimations and multi-literation to estimate nodes' locations, assuming an isotropic RF radio. The APIT positioning algorithm [4] is a scheme in which a node infers its position based on the possibility of being inside or outside of a triangle formed by any three anchors. Another set of solutions use Time of Arrival (TOA) [5] and Time Difference of Arrival (TDOA) [6] techniques to obtain pair-wise distances. These techniques demonstrate high accuracy in localization in real deployment. However, they require extensive infrastructure support.

The rest of the paper is structured as follows. In the second section we present system design and architecture of our Beidou navigation system aided method for localization in manually deployed wireless sensor networks. The implementation of the system is described in section three. We present our extensive experimental results. Conclusion and future works are presented in section four.

\section{SYSTEM DESIGN}

In this section, we present the design architecture of the system and describe the internals of software components. The system is divided into two software components: the Beidou navigation module and the sensor module. A deployment diagram is shown in Figure. 1.

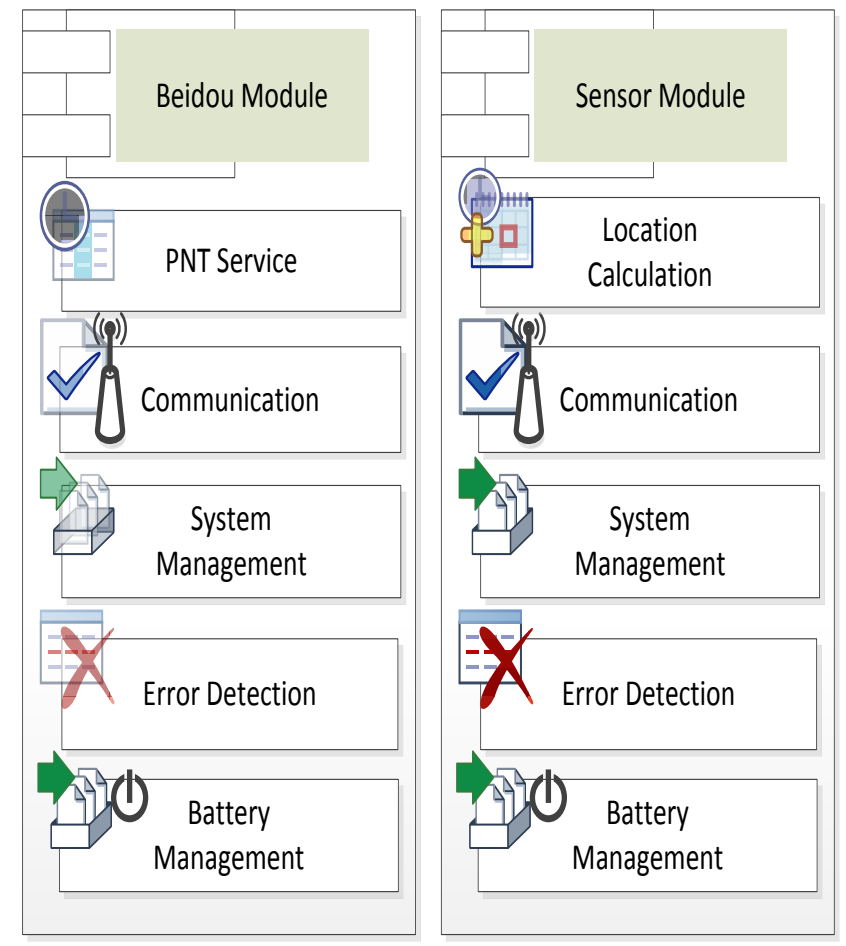

(a) Software components for Beidou navigation module. (b) Software components for sensor module.

Figure. 1 Software scheme for the system. 
The BeiDou navigation module runs on a DSP-based development board. The board is connected to a BeiDou navigation chip, and outputs its location information at periodic intervals. The sensor module component runs on all sensor nodes in the network. This component receives the location information broadcast by the Beidou navigation module and infers its position from the packets received. All complexity derived from the interaction with the PNT service is added to a single node (BeiDou navigation module). As a result, the size of the code and data memory used on the sensor node is significantly reduced and the costs are thus reduced.

Location information provided by Beidou navigation module is represented by the latitude and longitude, which are angular measures from the Equator to North or South, and Prime Meridian to East or West, respectively. Due to the relatively small size of a sensor network, the use of global coordinates is very inefficient. A local, Cartesian, coordinate system is derived. This local coordinate system of reference, which uses linear units, is better suited for WSN. The local system of reference has an origin specified in terms of global coordinates. Distance between this origin $(\lambda, \varphi)$ and another point, with a location $\left(\lambda_{i}, \varphi_{i}\right)$ can be computed as follows:

$$
d=\sqrt{\left(X \times\left(\varphi-\varphi_{i}\right)\right)^{2}+\left(Y \times\left(\lambda-\lambda_{i}\right)\right)^{2}}
$$

where,

$$
\begin{gathered}
X=\frac{\pi}{180}\left(\frac{a^{2} b^{2}}{\left(a^{2} \cos ^{2} \varphi+b^{2} \sin ^{2} \varphi\right)^{\frac{3}{2}}}+h\right) \\
Y=\frac{\pi}{180}\left(\frac{a^{2}}{\left(a^{2} \cos ^{2} \varphi+b^{2} \sin ^{2} \varphi\right)^{\frac{1}{2}}}+h\right) \cos \varphi
\end{gathered}
$$

The parameters in the above formulas are:

$$
\begin{aligned}
& a=6378.137 m \\
& b=6356752.3142 m
\end{aligned}
$$

$\mathrm{h}$ is the height over the ellipsoid.

The localization scheme has two distinct phases.

I. The first phase (Figure. 2.a) is during the deployment of the sensor nodes. The Beidou module periodically beacons its location; the sensor nodes that receive this beacon infer their location based on the information present in this beacon.

II. The second phase (Figure. 2.b) is during the system initialization. If at that time, a sensor node does not have a location; it will ask its neighbors for their location information. The location information received from neighbors is used to infer its position.

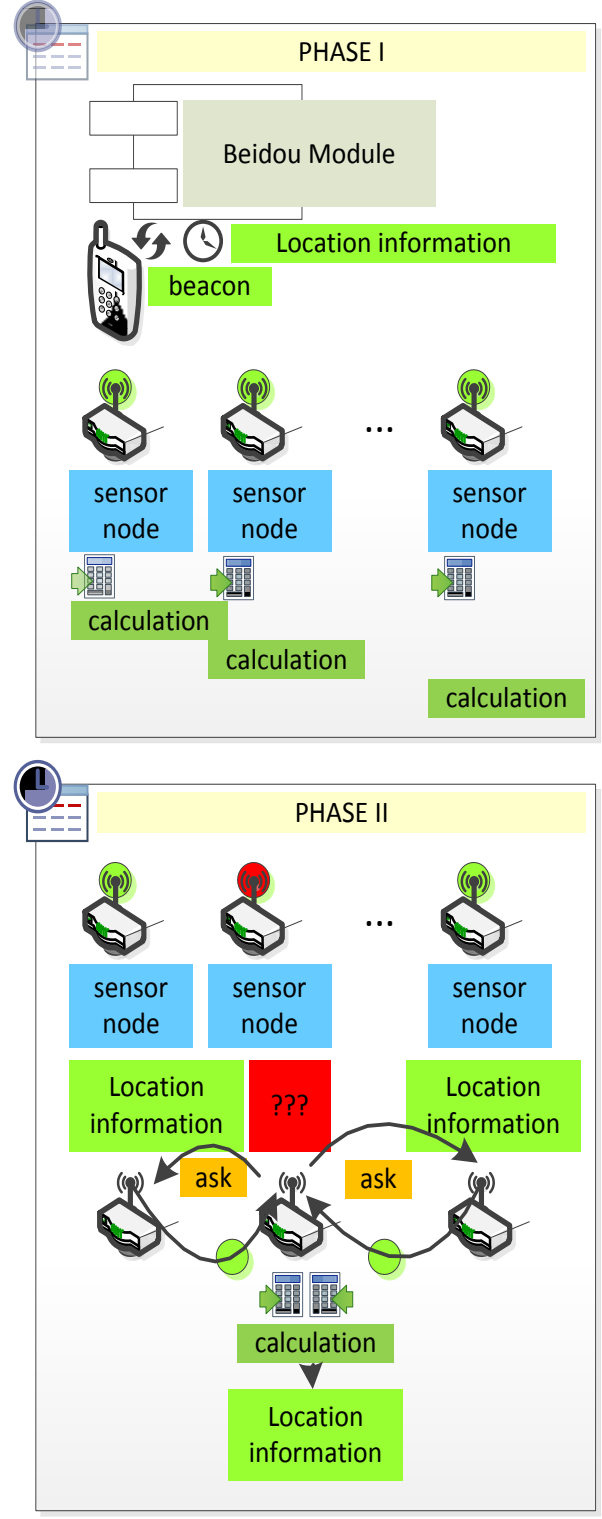

(a) Location infer in deployment phase (b) Location infer in initialization phase

Figure.2 The illustration on localization scheme.

\section{SYSTEM IMPLEMENTATION AND RESULTS}

\section{A. System Implementation}

The BeiDou navigation system aided method for localization in manually deployed wireless sensor networks requires that the BeiDou module has a BeiDou navigation chip attached to it. A BeiDou module without a reference point is in a development state. No messages are sent by the module, as long as it is in this state. A reference point can be obtained either through radio communication, or from flash memory. Once a reference point is obtained through radio, it is also stored in the flash memory and the module is in a initialized state, now. 


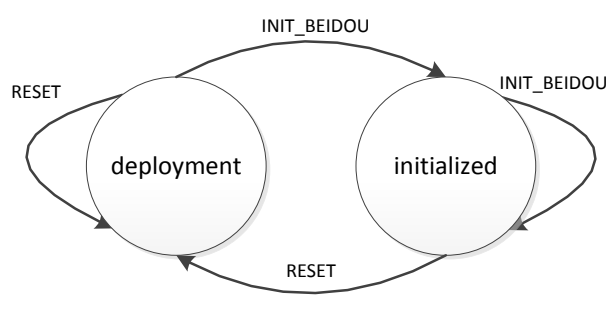

Figure.3 State Transformation diagram for Beidou module.

\begin{tabular}{|c|c|c|}
\hline $\begin{array}{c}\text { Origin } \\
\text { Latitude }\end{array}$ & $\begin{array}{c}\text { Origin } \\
\text { Longitude }\end{array}$ & $\begin{array}{c}\text { Sending } \\
\text { Period }\end{array}$ \\
\hline \hline 4 BYTEs & 4 BYTEs & 2 BYTEs \\
\hline
\end{tabular}

Figure. 4 INIT_LOCALIZATION packet formation.

The Beidou module sends location information by broadcasting an INIT_LOCALIZATION packet with a format shown in Figure. 4 and a prototype module is shown in Figure. 5 .

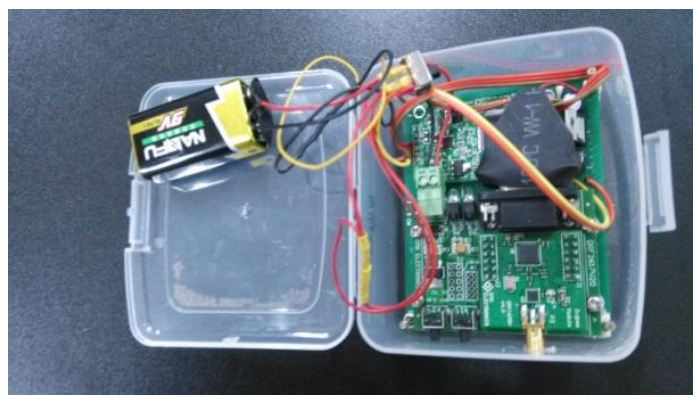

Figure. 5 Prototype system of the Beidou module.

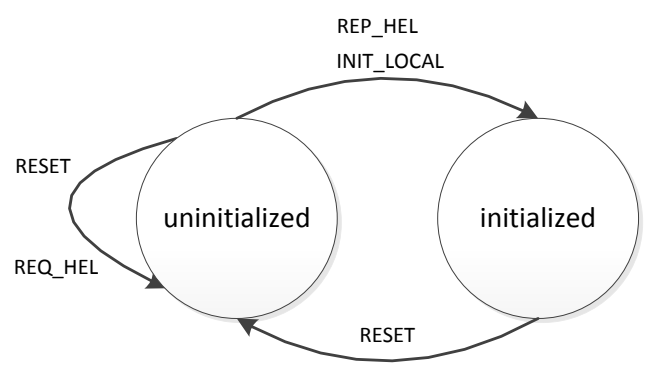

Figure.6 State Transformation diagram for sensor module.

The sensor module should be in one of two states: initialized (if location information is present) or uninitialized. The state transition diagram for it is shown in Figure.6.

A sensor module can become Initialized when: a) if it receives an INIT_LOCAL message, $b$ ) if the neighboring node of the sensor module sent a REP_HEL message.

If a sensor module enters the system initialization phase and it does not have a location, the sensor node broadcasts a packet of type REQ_HEL. Neighboring nodes that have a location respond by broadcasting packets of type REP_HEL. A sensor node that sent the message stores the responses in a buffer and computes its own location at the centroid of the locations received from its neighbors. A prototype sensor module is shown in Figure. 7.

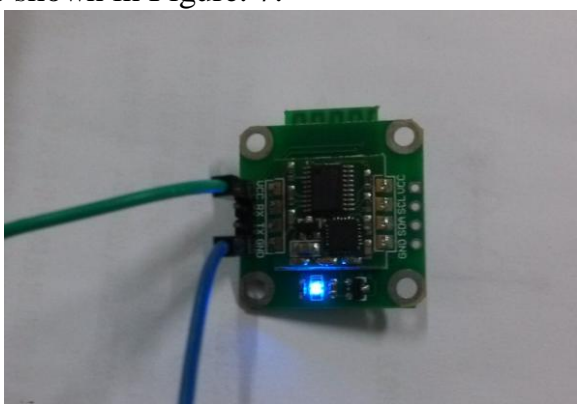

Figure. 7 Prototype system of the sensor module.

\section{B. Experimental Results}

The system was evaluated in an open field, as shown in Figure. 8. For an easier estimate of the localization error, we marked a grid mesh on the ground and deployed the sensors in grids.

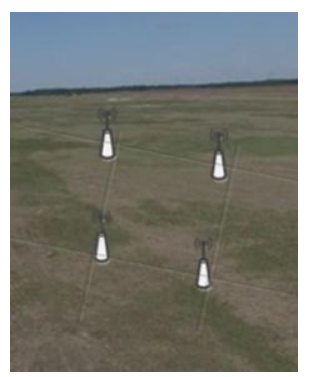

Figure. 8 Experimental deployment

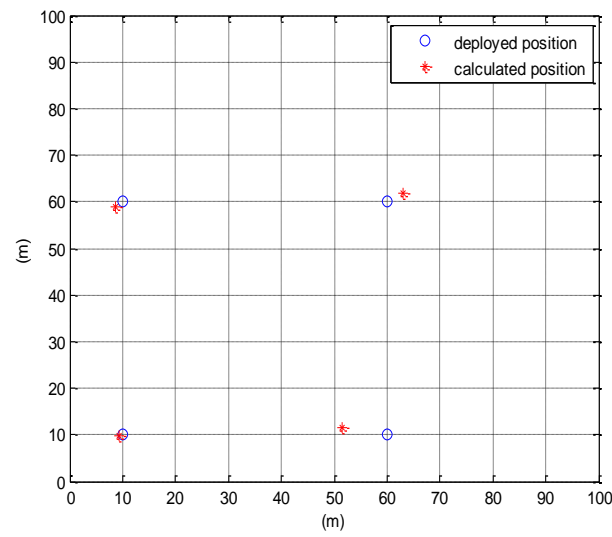

Figure.9 Result of the experimental evaluation.

The Beidou module for localization was carried during the walk when the sensor modules are deployed manually. The result of the experimental evaluation is shown in Figure. 9.

The average localization error is defined as:

$$
E=\frac{\sum_{i=1}^{4} \sqrt{\left(x_{i}-x_{i}^{\text {rrid }}\right)^{2}+\left(y_{i}-y_{i}^{\text {grid }}\right)^{2}}}{4}
$$

Thus, the average localization error is about 2 meters. 


\section{SUMMARY}

In this paper we presented the design, implementation and the evaluation of a localization solution that can be used in situations in which WSN are deployed manually. Experiments result show that $100 \%$ of the deployed notes have been successfully located and that the average errors are within 2 meters. The experience from the development of the current system can be further used in future research that will address the aerial deployment.

\section{REFERENCES}

[1] G. Lammel, J. Gutmann, L. Marti, M. Dobler, Indoor Navigation with MEMS sensors. Procedia Chemistry 1, (2009), 532-535.
[2] Zhi Shen, Jacques Georgy, Michael J. Korenberg, Aboelmagd Noureldin. Low cost two dimension navigation using an augmented Kalman filter/Fast Orthogonal Search module for the integration of reduced inertial sensor system and Global Positioning System. Transportation Research Part C 19(2011) 1111-1132.

[3] Haibo He, Jinlong Li, Yuanxi Yang, Junyi Xu, Hairong Guo, Aibing Wang Performance assessment of single and dual frequency BeioDou/GPS single-epoch kinematic positioning. GPS Solut (2014) 18: 393-403.

[4] Chen Pei. Attitude determination algorithms for spinning satellites using single antenna GPS receiver and MEMS gyro. Aerospace Science and Technology 26 (2013) 10-15.

[5] Saman Mukhtar Siddiqui, Fang Jiancheng. Navigation solution with Wavelet and Allan variance. Energy Procedia 11 (2011) 1727-1735.

[6] Johan Bijker, Willem Steyn. Kalman filter configurations for a low-cost loosely integrated inertial navigation system on an airship. Control Engineering Practice 16 (2008) 1509-1518. 